Check for updates

Cite this: RSC Adv., 2017, 7, 51426

Received 12th August 2017

Accepted 17th October 2017

DOI: 10.1039/c7ra08915e

rsc.li/rsc-advances

\section{Surface adsorption and thermodynamic properties of mixed system of ionic liquid surfactants with cetyltrimethyl ammonium bromide $\uparrow$}

\begin{abstract}
Li Qin and Xin-Hong Wang (D) *
The effects of temperature and concentration of CTAB added into imidazolium-based ionic liquids $\left(C_{n} \operatorname{mimBr}, n=10,12,16\right)$ solution were investigated, and surface active parameters and aggregation of these surfactants were explored by surface tension and conductivity measurements, which include critical micelle concentration (CMC), maximum surface excess concentration $\left(\Gamma_{\max }\right)$, minimum surface area per surfactant molecule $\left(A_{\min }\right)$, surface tension at the $C M C\left(\gamma_{C M C}\right)$, adsorption efficiency $\left(p C_{20}\right)$, and surface pressure $\left(\Pi_{\mathrm{CMC}}\right)$, as well as thermodynamic parameters of micellization. The results were reported in the presence of CTAB at $0,4,8,12$, and $16 \mathrm{mM}$ for $\mathrm{C}_{10} \mathrm{mimBr}, 0,0.2,0.4,0.8$, and $1.2 \mathrm{mM}$ for $C_{12} \mathrm{mimBr}$, and $0,0.04,0.08,0.12$, and $0.16 \mathrm{mM}$ for $C_{16} \mathrm{mimBr}$ in mixed systems. The increase of the CMCs with temperature were observed, while $p C_{20}, I_{\max }$, and standard entropy of aggregation were decreased, indicating that the aggregation is an enthalpy-driven process within the temperature limits of the present study. FT-IR and ${ }^{1} \mathrm{H}$ NMR measurements revealed that the changes mentioned above were controlled by the electrostatic effect and hydrophobic interactions between CTAB and ILs molecules.
\end{abstract}

\section{Introduction}

Ionic liquids (ILs) have gained great attention for their promising role as alternative media in catalysis, separation, and electrochemical processes owing to their unique chemical and physical properties, such as negligible vapor pressure, wide liquidous range, excellent solvation, high thermal stability, ionic conductivity and wide electrochemical windows. Especially, as an environmentally friendly solvent, also called a "green solvent", ILs make some contribution towards preventing environmental pollution by virtue of their non-volatility. Based on their inherent amphiphilic structure consisting of a non-polar hydrophobic tail and a polar cationic head group, a typical long-chain ILs could possess surface active properties similar to those of conventional cationic surfactants, so they have been extensively studied in the field of colloid and interface science in recent years. For instance, the surface tension and critical micelle concentration (CMC) of long-chain ILs decrease with alkyl chain length. ILs applications in wide fields are closely dependent on such self-assembly behavior. ${ }^{1}$ In addition, ILs with different cations and anions have been

Jiangsu Key Laboratory for Bioresources of Saline Solis, School of Chemistry and Environmental Engineering, Yancheng Teachers University, Xiwang Road, Yancheng 224051, Jiangsu Province, P. R. China. E-mail: wangxhong1227@163.com; Fax: +86 515 88233187; Tel: +86 51588233187

$\dagger$ Electronic supplementary information (ESI) available. See DOI: 10.1039/c7ra08915e reported to understand the effect of the ions on aggregation behavior. ${ }^{2-4}$ However, it is unclear how the surface active and micellization features depend upon their molecular characteristics, concentration, temperature and additives etc. In view of this, study of the interaction and microstructural characteristics of micellar systems is always an important subject area in the discipline of colloid and interface science.

Numerous pioneering studies on multicomponent mixtures of ionic liquid have widened the application field of ILs. For instance, phase behaviour of aqueous two-phase systems containing an ionic liquid, ${ }^{5}$ molecular interactions and preparation of nanoparticles, ${ }^{6,7}$ and effects on properties of nanocomposites. ${ }^{8}$ In these studies, the binary mixture is relatively fascinating from an academic point of view wherein including common ionicionic systems, ${ }^{9-11}$ and ionic-nonionic systems. ${ }^{12-14}$ The CTAB/ bmim-octylSO ${ }_{4}$ binary system in aqueous solution at two total concentrations and different $\mathrm{CTAB}$ mole fractions was investigated and found to form the CTA-octylSO ${ }_{4}$ catanionic complex, ${ }^{9}$ which is considered analogue to the effect of the addition of inorganic salts in such similar systems. ${ }^{15}$ The aggregation behaviour of the alkyl triphenylphosphonium bromide $\left(\mathrm{C}_{n} \mathrm{TPPBr}\right.$, $n=10,12,14,16)$ /Triton X-100 system was studied by Thakkar et al. ${ }^{13}$ Furthermore, a generalized phase separation model was discussed in ionic-nonionic mixtures. ${ }^{16}$ The ionic liquid TEA $\left(\mathrm{BF}_{4}\right)$ and non-ionic surfactant TX-100/Brij-56/Brij-58/Tween20/Tween-80 in aqueous media at different temperatures were studied, respectively. ${ }^{17}$ Some other reports have just been published regarding the IL surfactant applications. ${ }^{18,19}$ Different 
measurement technologies were used to obtain the needed surface activities and thermodynamics functions, such as a small-angle neutron scattering (SANS), ${ }^{13,20}$ dynamic light scattering (DLS) and viscosity measurements, ${ }^{17}$ conductivity, ${ }^{12,21,22}$ surface tension measurements, ${ }^{23}$ as well different analytical methods, FT-IR, ${ }^{3}$ UV-visible spectrum, ${ }^{22,24}$ fluorescence spectroscopy, ${ }^{4,22,25,26}$ isothermal titration calorimeter ${ }^{25}$ and NMR technology. 27,28

Apart from the self-assemblies and applications of ILs surfactants in various fields, the aggregation behavior of 1-alkyl3-methylimidazolium $\left(\mathrm{C}_{n} \mathrm{mim}^{+}\right)$in water has been presented by many research groups. ${ }^{13,15,29}$ The micellization behavior of the $\mathrm{DTAB} / \mathrm{bdmimCl}$ system has been reported by Pal' group, ${ }^{27}$ also including their related work on tetradecyltrimethylammonium bromide $\mathrm{TTAB} / \mathrm{C}_{5} \mathrm{mim} / \mathrm{PF}_{6}$ system, ${ }^{30} \mathrm{CTAB} / \mathrm{bdmimBr}$ system, ${ }^{31}$ odmimCl/DTAB mixed system, ${ }^{21}$ and TTAB/bdmimBr mixture. ${ }^{28}$ However, in all these systems, all studied ILs as an additive were added into traditional surfactant solution to form IL-surfactant mixtures. The CMC values of the mixtures increase with increasing wt $\%$ IL content and temperature at the same IL concentration. So it would be interesting to observe the effect of $\mathrm{CTAB}$ as an additive on the micellization of IL as no reports have been found to date. Consequently the purpose of the present work is to study the effects of temperature and CTAB addition into ILs $\left(\mathrm{C}_{n} \operatorname{mimBr}, n=10,12,16\right)$ solution on the surface properties of the mixture system and the thermodynamic properties of micellization. This prompted us to carry out systematic investigations with a focus mainly on examining the surface activity and aggregate formation of ionic liquids based on these long-chain ILs and to assess the effect of chain length and head group nature on the CMC, $p C_{20}, \Gamma_{\max }, A_{\min }$, and thermodynamic parameters of aggregation by surface tension and electrical conductance measurements for mixed systems. Conductivity and surface tension measurements were used to obtain the CMC values for three IL/CTAB solutions, and the thermodynamic parameters were used to analyze the driving force of micellization. In addition, ${ }^{1} \mathrm{H}$ NMR and FT-IR measurements were done to understand the interactions between the IL and the surfactant molecules. The results obtained could be compared with similar systems for further applications of binary systems in some areas of separation, adsorption, and other surface research fields.

\section{Materials and methods}

\subsection{Materials}

Cetyltrimethyl ammonium bromide (CTAB, MW: 364.45 , purity $\geq 99 \%$, CAS number: 57-09-0) was obtained from Sinopharm Chemical Reagent Co., Ltd (Shanghai, China). 1-Hexadecyl-3methylimidazolium bromide $\left(\mathrm{C}_{16} \mathrm{mimBr}\right.$, MW: 387.44 , purity $\geq$ 99.9\%, CAS: 132361-22-9), 1-dodecyl-3-methylimidazolium bromide $\left(\mathrm{C}_{12} \mathrm{mimBr}, \mathrm{MW}: 331.33\right.$, purity $\geq 99.9 \%$, CAS: 61546-00-7), and 1-decyl-3-methylimidazolium bromide $\left(\mathrm{C}_{10} \mathrm{mimBr}\right.$, MW: 303.28 , purity $\geq 99.9 \%$, CAS: 188589-32-4) were purchased from Shanghai Cheng Jie Chemical Co., Ltd., China, and these ILs were dried at $343 \mathrm{~K}$ for $24 \mathrm{~h}$ in vacuum drying oven (DZF-6050, Shanghai Shupei Laboratory Equipment
Co., Ltd., China) prior to use. The molecular structures of the ILs and CTAB are shown in Fig. 1. $\mathrm{D}_{2} \mathrm{O}(D>99.96 \%)$ was obtained from Sigma-Aldrich and used as the solvent in ${ }^{1} \mathrm{H}$ NMR measurements.

\subsection{Methods}

2.2.1 Conductivity measurement. The electrical conductivities were measured at different temperatures using a digital conductivity meter (DDSJ-308A, Shanghai Leici Instrument Factory, China) equipped with a platinized platinum electrode. A stable temperature was maintained with a super-thermostatic water bath (ZC-18Q, Tianheng Instrument Factory, Ningbo, China). Before the measurements, the conductivity cell constant was calibrated with a known specific conductance aqueous solution of $0.01 \mathrm{M}$ potassium chloride aqueous solutions. At least three measurements were made every time and the specific conductivity was calculated from the mean value. The conductance due to water ionization $\left(5.83 \mu \mathrm{s} \mathrm{cm}^{-1}, \mathrm{pH}=6.98\right)$ was subtracted from the measured data before data analysis. The uncertainty in conductivity measurements was less than $\pm 0.4 \%$. The cell assembly was dipped in glass vials kept in a water bath thermostatted at the studied temperature maintained to $\pm 0.1 \mathrm{~K}$.

2.2.2 Surface tension measurement. The surface tension for aqueous solutions of single surfactants and binary mixtures were assessed using the maximum bubble pressure method with a tensiometer (DP-AW-1, Nanjing SangLi Electronic Equipment Factory, China) under atmospheric pressure. The calibration was performed by measuring the surface tension of doubly distilled de-ionized water $\left(71.97 \mathrm{mN} \mathrm{m}^{-1}\right)$ before each experiment. The temperature was maintained within $\pm 0.1 \mathrm{~K}$ by circulating water. The stock solutions were then diluted with fresh distilled water to desired concentrations. The uncertainty in the measured surface tensions was within $\pm 0.03 \mathrm{mN} \mathrm{m}^{-1}$.

The surface excess adsorption, $\Gamma_{\max }$, and the minimum area per surfactant molecule, $A_{\min }$, at the air-solvent interface were calculated using surface tension measurement values according to the Gibbs adsorption isotherm equation, given by eqn (1) and (2):

$$
\begin{gathered}
\Gamma_{\max }=-\frac{1}{R T}\left(\frac{\partial \gamma}{\partial \ln c}\right)_{T} \\
A_{\min }=\frac{1}{L \Gamma_{\max }}
\end{gathered}
$$

where $R$ is the gas constant, $T$ is the absolute temperature, $\gamma$ is the surface tension, and $c$ is the IL concentration of mixed solutions. $L$ is Avogadro's constant. The adsorption efficiency $\left(p C_{20}\right)$ is an important surface activity parameter, which is defined by eqn (3):

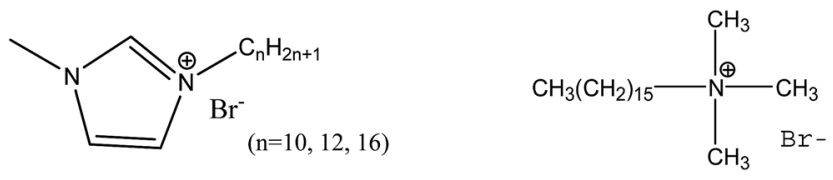

Fig. 1 Molecular structures of ILs and CTAB 


$$
p C_{20}=-\log C_{20}
$$

Surface pressure at the $\mathrm{CMC}, \Pi_{\mathrm{CMC}}$, is a measure of the efficiency of the surfactant to lower the surface tension of the water, and is obtained by means of eqn (4):

$$
\Pi_{\mathrm{CMC}}=\gamma_{0}-\gamma_{\mathrm{CMC}}
$$

where $C_{20}$ is the effective concentration of all surfactants in the mixed system required to reduce the surface tension of pure water by $20 \mathrm{mN} \mathrm{m}^{-1} \gamma_{0}$ is the surface tension of pure water for the respective temperature, and $\gamma_{\mathrm{CMC}}$ is the measured surface tension at the CMC.

The enthalpic contribution to the micellization process, $\Delta H_{\mathrm{m}}$, was determined based on $\Delta G_{\mathrm{m}}$, using the Gibbs-Helmholtz relation as shown in eqn (5) and (6). The standard entropy of the micellization, $\Delta S_{\mathrm{m}}$, was calculated from eqn (7):

$$
\begin{gathered}
\Delta G_{\mathrm{m}}=(2-\alpha) R T \ln X_{\mathrm{CMC}} \\
\Delta H_{\mathrm{m}}=-R T^{2}\left[(2-\alpha) \frac{\mathrm{d} \ln X_{\mathrm{CMC}}}{\mathrm{d} T}+\ln X_{\mathrm{CMC}} \frac{\mathrm{d}(2-\alpha)}{\mathrm{d} T}\right] \\
\Delta S_{\mathrm{m}}=\frac{\Delta H_{\mathrm{m}}-\Delta G_{\mathrm{m}}}{T}
\end{gathered}
$$

where $X_{\mathrm{CMC}}$ is the CMC in mole fraction, the degree of dissociation, $\alpha$, was associated to the degree of ionization, which represents the fraction of amphiphile ions in the aggregate neutralized by counter anions. $R$ is the gas constant, and $T$ is temperature. According to eqn (5), $\Delta G_{\mathrm{m}}$ of micellization at different temperatures may be calculated using $X_{\mathrm{CMC}}$. Thus, the curve of $\Delta G_{\mathrm{m}} / T$ against $1 / T$ can be drawn, and $\Delta H_{\mathrm{m}}$ can be finally calculated from the slopes of tangential lines at various temperatures. The parameters thus obtained are presented in Table 2 .

2.2.3 FT-IR spectra measurement. The spectra of the samples were measured using a Thermo Scientific Nicolet IS10 FT-IR spectrometer equipped with a standard $\mathrm{KBr}$ beam splitter and DTGS detector. The mixture spectra of the $\left[\mathrm{C}_{n}\right.$ mimBr$] /$ CTAB solution were scanned on the Smart iTR ${ }^{\mathrm{TM}}$ diamond ATR reflection accessory for qualitative analysis in this study. The spectra from samples (pure IL or mixture of IL and TX-100 without water) were collected in 40 seconds over the full mid-infrared range (4000 to $400 \mathrm{~cm}^{-1}$ ). The samples needed to be premixed (molar ratio $1: 1$ ) for a period of time before analysis. Instrument control, data collection and analysis were performed using the Thermo Scientific OMNIC software package.

2.2.4 ${ }^{1}$ H NMR measurements. The NMR chemical shifts for various protons were observed with a Bruker AVANCE III HD $400 \mathrm{M}$ NMR spectrometer at room temperature (about $298 \mathrm{~K}$ ). Deuterium oxide $\left(\mathrm{D}_{2} \mathrm{O}\right)$ as a solvent for all the NMR measurements was used to determine chemical shift $\delta$ for the $\left[\mathrm{C}_{n^{-}}\right.$ $\operatorname{mimBr}] /$ CTAB solution.

All the experiments were carried out in doubly distilled deionized water.

\section{Results and discussion}

\subsection{Conductivity measurement}

3.1.1 CMC determination. Conductivity measurements were carried out in solutions of ionic liquids over a wide concentration range at $298.15-338.5 \mathrm{~K}$ to obtain CMC values with the addition of the surfactant CTAB and without CTAB. Sufficient care was taken to avoid high IL concentrations to ensure that the solutions were not highly viscous and were free of air bubbles during the measurements.

For pure ionic liquids solutions, the plots of conductivity against concentration of IL surfactant (Fig. 2) displayed the difference of the curves well, namely the slope in the premicellar region was higher than one in post-micellar region regardless of the pure IL surfactants, or mixture solutions. The presence of turning point is attributable to the change of aggregation state from CTAB molecules and IL molecules. Thus, the abscissa value corresponding to the turning point is the CMC value of the studied system, as listed in Table 1. Reasonably, the CMC decreased with increasing length of alkyl chain and such a systematic decrease could be due to the enhanced hydrophobic character of higher alkyl chains. ${ }^{2}$ Therefore, it can be concluded that the interfacial activity of the pure IL surfactants is in the order of $\mathrm{C}_{16} \mathrm{mimBr}>\mathrm{C}_{12} \mathrm{mimBr}>\mathrm{C}_{10} \mathrm{mimBr}$. It is therefore suggested that the aggregation process of long-chain ILs in water needs to be close to that of the micellization process of classical ionic surfactants in aqueous media. Furthermore, the degree of dissociation $(\alpha)$ for these systems was obtained from the ratio of the slopes of the two linear lines drawn through regions corresponding to above and below the CMC value, as listed in Table 1.

Considering the CMC of CTAB is about $0.90 \mathrm{mM}, 9,27$ the concentrations values of $\mathrm{CTAB}$ that lie on both sides of the CMC of $\mathrm{CTAB}$ were selected to investigate the effect of adding CTAB monomer or micelles to IL aqueous solution on the CMC values and surface activities of mixed systems. The representative plots of the conductance of the mixed system $\left(\mathrm{C}_{12}\right.$ mimBr/CTAB system) versus IL concentration on addition of different concentrations of CTAB in aqueous medium are shown in Fig. 3, while conductivity measurement results for $\mathrm{C}_{10} \mathrm{mimBr} /$ CTAB and $\mathrm{C}_{16} \mathrm{mimBr} / \mathrm{CTAB}$ are depicted in Fig. $\mathrm{S} 1$ and $\mathrm{S} 2$ $(\mathrm{ESI} \dagger)$ and the measured data are given in Table 1. A similar change to that observed in Fig. 3 can be found and the CMCs of the mixed systems can be calculated.

According to the data from Fig. 3, S1 and S2, $\dagger$ the CMCs, the degree of dissociation $(\alpha)$ and the thermodynamic parameters of aggregation for ILs/CTAB in water were calculated at five different temperatures of $298.15,308.15,318.15,328.15$ and $338.15 \mathrm{~K}$. The corresponding data are listed in Table 1 . The data from Table 1 show that the CMCs value of the $\mathrm{C}_{n} \mathrm{mimBr}$ aqueous systems decreased with the increase in the alkyl chain length and increased with increasing temperature, ${ }^{27,32}$ which is consistent with the description in the literature ${ }^{33}$ and the results for other IL solutions. ${ }^{25}$ This can be interpreted as elevated temperature causes the disruption of the water structure leading to low solubility of the surfactant. Besides, the elevated 

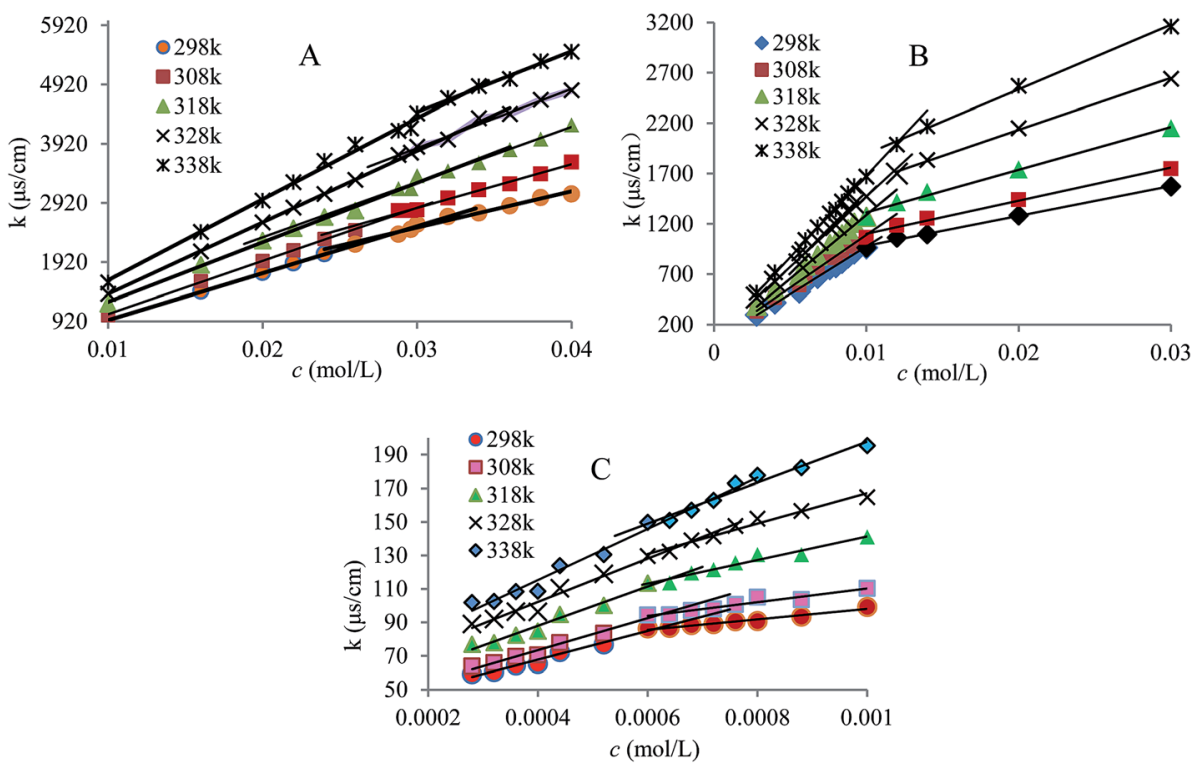

Fig. 2 Conductivity of pure $\mathrm{C}_{10} \operatorname{mimBr}(\mathrm{A}), \mathrm{C}_{12} \mathrm{mimBr}(\mathrm{B})$ and $\mathrm{C}_{16} \mathrm{mimBr}(\mathrm{C})$ in aqueous solutions at different temperatures.

temperature causes difficulties in forming the micelles, mainly due to the hydration of the ion being greatly enhanced, which significantly weakens the electrostatic interaction of micellization.

In addition, the CMCs of the $\mathrm{C}_{n}$ mimBr/CTAB aqueous systems decline with increasing $\mathrm{CTAB}$ concentration at the same temperature. Although the positive charges on the head group of CTAB and the imidazolium ring of the IL repel each other, in fact, electrostatic attraction effect between the counterion of the IL, $\mathrm{Br}^{-}$, and the head group of the CTAB molecules may reinforce micelle formation to make the CMC value low or the surface activity high. At the same time, the hydrophobic

Table 1 Thermodynamic functions of $\mathrm{C}_{n} \mathrm{mimBr}$ in the absence of CTAB (conductivity method) ${ }^{a}$

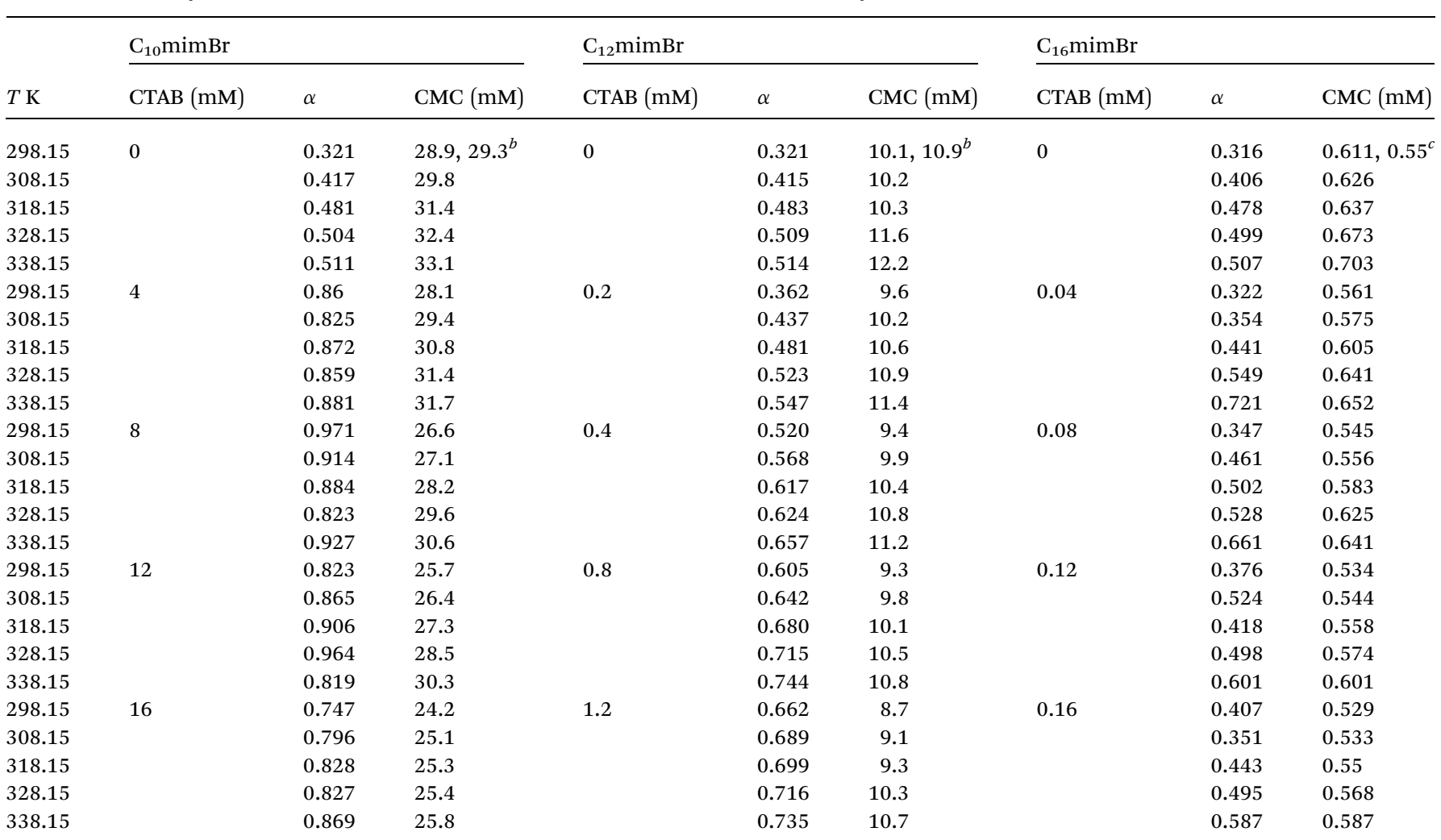

${ }^{a}$ Standard uncertainties $s$ are $s(\mathrm{CMC})= \pm 0.03(\mathrm{mM}) .{ }^{b}$ The value is from the literature. ${ }^{25}{ }^{c}$ The value is from the literature. ${ }^{4}$ 

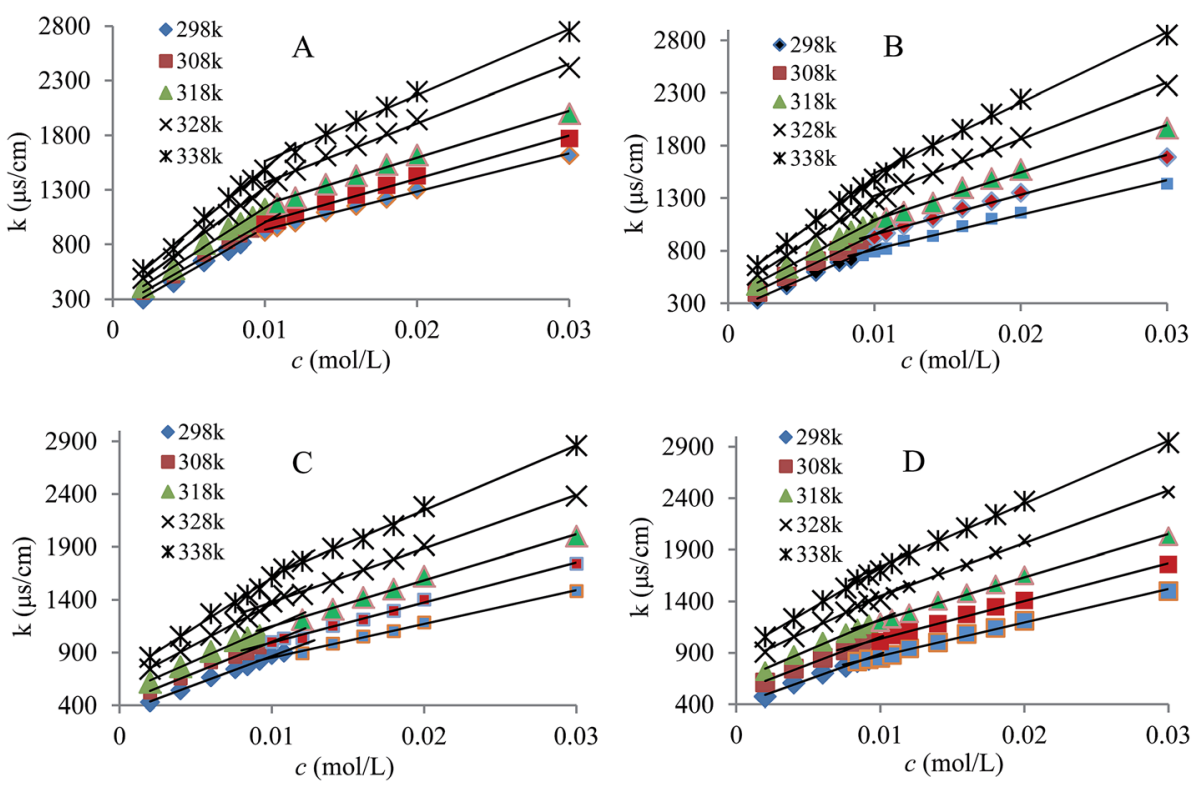

Fig. 3 Conductivity of the mixed system of $C_{12}$ mimBr/CTAB at different temperatures. $C_{C T A B}(A-D): 0.2,0.4,0.8$ and $1.2 \mathrm{mM}$.

interaction between the tail of the CTAB and the alkyl chain of the IL cation promote the procession of micellization. This finding differs from the $\mathrm{CTAB} / \mathrm{bmim}$-octylSO ${ }_{4}$ system reported by other authors, ${ }^{9}$ who thought the formation of the pseudodouble chain catanionic surfactant (CTA-octylSO ${ }_{4}$ ) led to a much lower critical aggregation concentration (CAC) and surface tension value at the CAC for the mixtures than for the individual components, whereas similar results in CTAB/SDS system have been obtained showing that the electrostatic repulsion of the head groups decreases and consequently the CMC is reduced when SDS gets into the solution. ${ }^{34}$ This behavior is also similar to the effect of electrolytes such as sodium chloride $(\mathrm{NaCl})$ when added to surfactant solutions. Liu et al. found that the $\mathrm{CMC}$ values of $\mathrm{C}_{12} \mathrm{mimBr}$ aqueous solution decreased with increasing $\mathrm{NaBr}$ concentration, and illustrated that the phenomenon arises from the reduction of electrostatic repulsion between the surfactant head groups caused by the additional ions in the solution. ${ }^{15}$ Additionally, the degree of dissociation $(\alpha)$ decreased with the increase in the alkyl chain length. The small value of $\alpha$ in general indicates that the aggregate structures are compact for $\mathrm{C}_{n} \mathrm{mimBr} / \mathrm{CTAB}$ aqueous systems in water.

3.1.2 Thermodynamic functions. The temperature dependence of the CMC has been employed to compute the thermodynamic parameters of micellization in order to obtain better knowledge about the behavior of the surfactant and intermolecular interactions present in such systems. ${ }^{35}$ The thermodynamic parameters of the $\mathrm{C}_{n} \mathrm{mimBr} / \mathrm{CTAB}$ mixed system at different CTAB concentrations are shown in Fig. 4. The standard free energy change of the micelle formation $\left(\Delta G_{\mathrm{m}}\right)$ revealed a rather slight decrease with the increment of temperature in mixed systems. There is no doubt that the negative values of $\Delta G_{\mathrm{m}}$ indicated the spontaneous micellization. The more negative value of $\Delta G_{\mathrm{m}}$ with the increase in the length of the alkyl chain is attributed to the fact that the longer alkyl chains facilitate the spontaneous aggregate formation. Furthermore, the negative $\Delta H_{\mathrm{m}}$ implies that micellization formation remains mainly an exothermic process, which is owing to greater dissociation of counter-ions from the micellar surface leading to a more exothermic micellization process. However, $\Delta H_{\mathrm{m}}$ firstly increased and then declined with temperature limits of 298.15-338.15 K with CTAB concentrations of $0.2,0.4,0.8$ and $1.2 \mathrm{mM}$, as can be seen from Fig. 4.

Meanwhile, entropy change $\left(-T \Delta S_{\mathrm{m}}\right)$ was plotted against temperature to better understand the contribution of entropy to the free energy (Fig. 4). It is noteworthy that the trend in the change of $-T \Delta S_{\mathrm{m}}$ with temperature in the mixed solution is analogous to $\Delta H_{\mathrm{m}}$. It can be seen from Fig. $4 \mathrm{~A}-\mathrm{D}$ that $-T \Delta S_{\mathrm{m}}$ $\left(\Delta S_{\mathrm{m}}\right)$ gradually decreases (increases) with temperature at the same low CTAB concentration of 0.2 and $0.4 \mathrm{mM}$, then

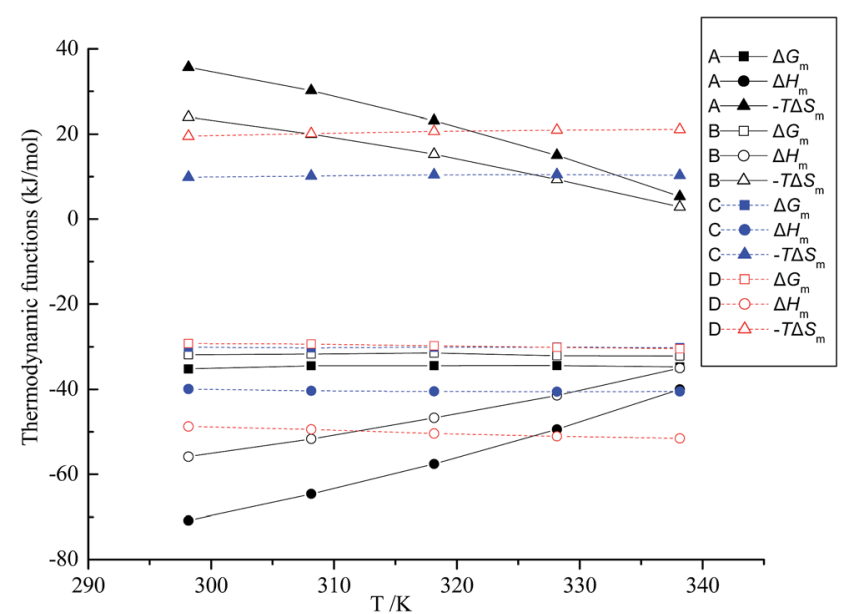

Fig. 4 Thermodynamic functions $\left(\mathrm{kJ} \mathrm{mol}^{-1}\right)$ of $\mathrm{C}_{12} \mathrm{mimBr} / \mathrm{CTAB}$ system against temperature. $C_{C T A B}(A-D): 0.2,0.4,0.8$ and $1.2 \mathrm{mM}$. 
substantially keeps flat at $0.8 \mathrm{mM}$, and finally increases when reaching $1.2 \mathrm{mM}$. Obviously, this change may be dependent on the presence of the CTAB molecules in Fig. 4A-D with the same IL concentration. ${ }^{17}$ For CTAB monomer $\left(c_{\mathrm{CTAB}}<0.9 \mathrm{mM}\right)$ added into IL solution, the disruption of the water structure by the interaction between IL molecules and CTAB monomer makes the arrangement of the mixture system less and less ordered. However, $\Delta S_{\mathrm{m}}$ decreases with temperature when CTAB micelles $\left(c_{\mathrm{CTAB}}>0.9 \mathrm{mM}\right)$ are added into IL solution. The essence of this phenomenon is that the higher temperature makes it more difficult to dissolve nonpolar substances in water, leading to enhancement of hydrophobic interactions between micelle molecules. In other words, hydrophobic alkyl chains come closer to each other in the micellar phase. As a result, the molecular arrangement is more ordered so that entropy is reduced. According to the data in Table 1, the CMCs of the two surfactant mixtures at elevated temperatures were lower than each surfactant, which also gives evidence of the strong synergism produced in mixed aggregates. ${ }^{34}$ In general, Fig. 4 shows that the contribution of enthalpy term $\Delta H_{\mathrm{m}}$ to $\Delta G_{\mathrm{m}}$ is superior to $\left(-T \Delta S_{\mathrm{m}}\right)$ at all studied temperatures. Therefore, it can also be concluded that the micelle formation of the $\mathrm{CTAB} / \mathrm{C}_{n} \mathrm{mimBr}$ mixture is an enthalpy-driven process. The critical micelle concentration (CMC) determined from conductivity data was also found in the study of the CTAB/DTAB-glycyl dipeptide system, ${ }^{36}$ which revealed a decrease in the CMC values for both the surfactants with [glycyl dipeptide] and demonstrated the entropy-enthalpy contribution to the micellization process.

\subsection{Surface tension measurement}

3.2.1 CMC determination. Surface tension measurements were performed at different temperatures. Based on the plots of surface tension versus ILs concentration depicted in Fig. 5-7, several different parameters related to the surface activities are listed in Table 2 wherein the CMC value of the pure ILs are from the previous work. ${ }^{37}$ It was obvious that the surface tensions of the mixed systems in aqueous media exhibited a steep decrease

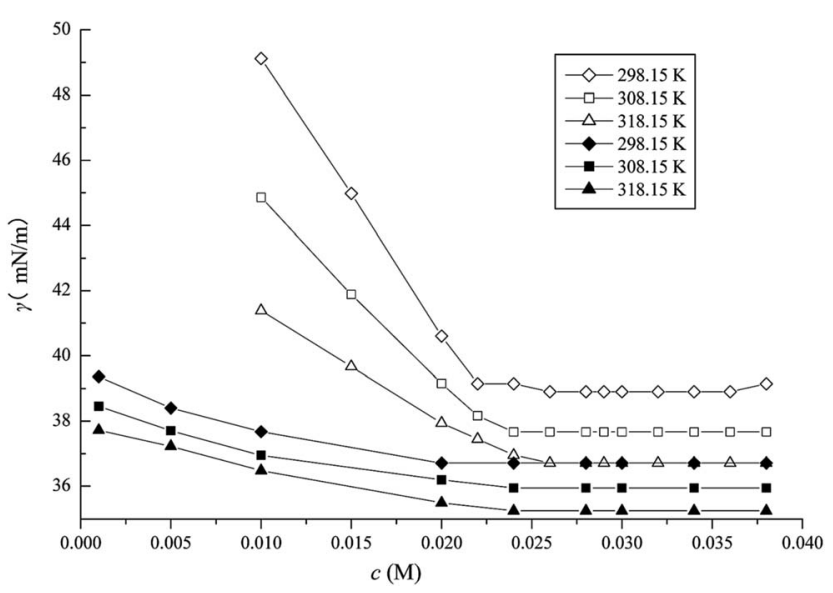

Fig. 5 Surface tension of $\mathrm{C}_{10} \mathrm{mimBr}$ in the presence of $C T A B$ $(\diamond 14 \mathrm{mM}, 298.15 \mathrm{~K}),(\square 14 \mathrm{mM}, 308.15 \mathrm{~K}),(\triangle 14 \mathrm{mM}, 318.15 \mathrm{~K})$, $(\diamond 30 \mathrm{mM}, 298.15 \mathrm{~K}),(\square 30 \mathrm{mM}, 308.15 \mathrm{~K})$ and $(\Delta 30 \mathrm{mM}, 318.15 \mathrm{~K})$.

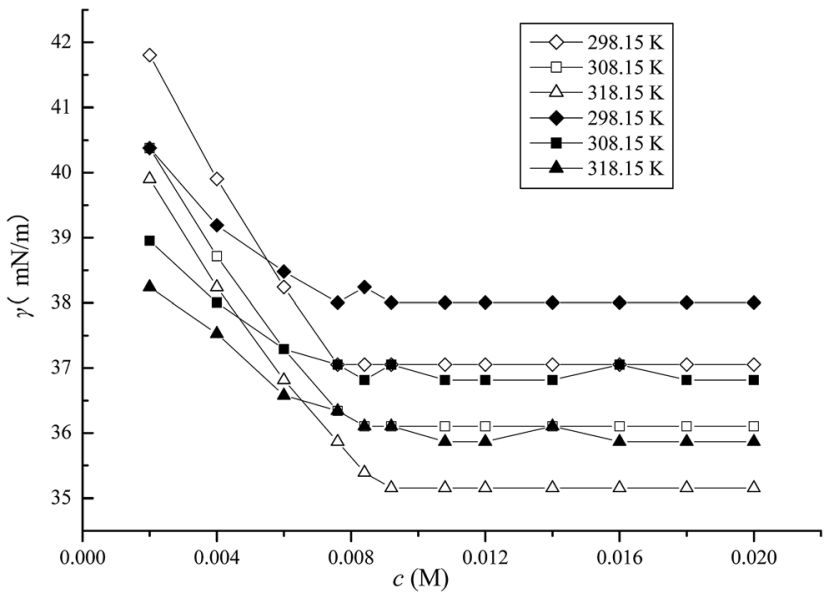

Fig. 6 Surface tension of $\mathrm{C}_{12} \mathrm{mimBr}$ in the presence of CTAB ( $\diamond 2 \mathrm{mM}$, $298.15 \mathrm{~K}),(\square 2 \mathrm{mM}, 308.15 \mathrm{~K}),(\triangle 2 \mathrm{mM}, 318.15 \mathrm{~K}),(\diamond 8 \mathrm{mM}, 298.15 \mathrm{~K})$, $8 \mathrm{mM}, 308.15 \mathrm{~K})$ and $(\boldsymbol{\Delta} 8 \mathrm{mM}, 318.15 \mathrm{~K})$.

with an increase of IL concentration up to a certain concentration and subsequently remained constant. The CMC value was obtained by the intersection between the rapidly decreasing line and the flat line of surface tension. As shown in Fig. 5-7, in the case of CTAB at $14 \mathrm{mM}$ or $30 \mathrm{mM}$ in the $\mathrm{C}_{10} \mathrm{mimBr} / \mathrm{CTAB}$ system, a dramatic decrease of the surface tension was observed compared with a moderate decrease at $2 \mathrm{mM}$ or $8 \mathrm{mM}$ CTAB in the $\mathrm{C}_{12} \mathrm{mimBr} / \mathrm{CTAB}$ system, and $0.02 \mathrm{mM}$ or $0.12 \mathrm{mM}$ in the $\mathrm{C}_{16} \mathrm{mimBr} / \mathrm{CTAB}$ system. These trends in the $\mathrm{CMC}$ value associated to temperature and $\mathrm{CTAB}$ concentration endorse the results drawn from the conductivity method. Chauhan et al. applied surface tension to investigate $\mathrm{CM}(\mathrm{A}) \mathrm{C}$ of a ternary (water + lactose $+\mathrm{NaC} / \mathrm{NaDC}$ ) system. ${ }^{26}$ The decrease of the CM(A)C values of bile salts in aqueous lactose solution was found to be in excellent agreement with the mixture systems in this work. Moreover, they employed conductivity and UV-vis probe to examine the interactions of the ionic liquid

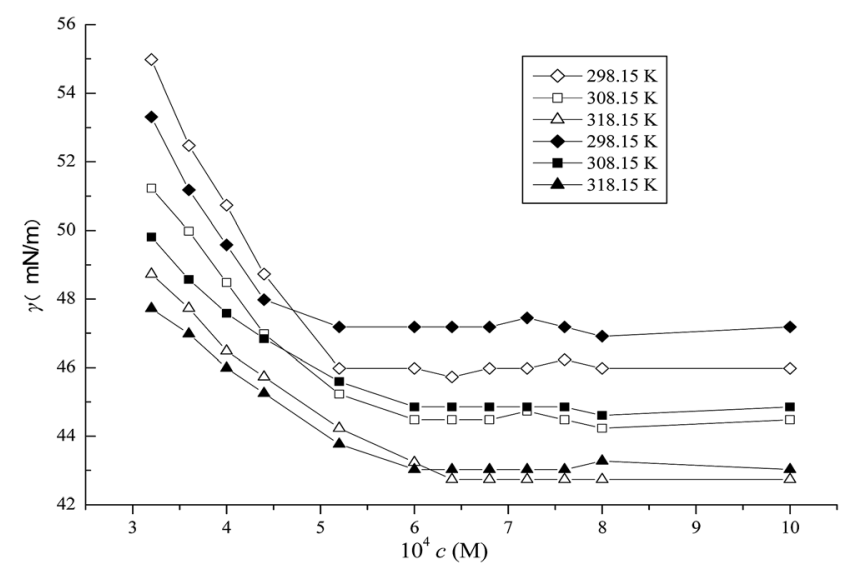

Fig. 7 Surface tension of $\mathrm{C}_{16} \mathrm{mimBr}$ in the presence of CTAB ( $\diamond$ $0.02 \mathrm{mM}, 298.15 \mathrm{~K}),(\square 0.02 \mathrm{mM}, 308.15 \mathrm{~K}),(\triangle 0.02 \mathrm{mM}, 318.15 \mathrm{~K})$, $(\diamond 0.12 \mathrm{mM}, 298.15 \mathrm{~K}),(\boldsymbol{\square} 0.12 \mathrm{mM}, 308.15 \mathrm{~K})$ and $(\boldsymbol{\Delta} 0.12 \mathrm{mM}$, $318.15 \mathrm{~K})$. 
Table 2 Thermodynamic functions of $\mathrm{C}_{n} \mathrm{mimBr}$ in the absence of CTAB (surface tension method) ${ }^{a}$

\begin{tabular}{|c|c|c|c|c|c|c|c|c|c|c|}
\hline CTAB (mM) & $T \mathrm{~K}$ & $\mathrm{CMC}(\mathrm{mM})$ & $\begin{array}{l}\Delta G_{\mathrm{m}} \\
\left(\mathrm{kJ} \mathrm{mol}^{-1}\right)\end{array}$ & $\begin{array}{l}\Delta H_{\mathrm{m}} \\
\left(\mathrm{kJ} \mathrm{mol}^{-1}\right)\end{array}$ & $\begin{array}{l}-T \Delta S_{\mathrm{m}} \\
\left(\mathrm{kJ} \mathrm{mol}{ }^{-1}\right)\end{array}$ & $\begin{array}{l}\Gamma_{\max } \\
\left(\mu \mathrm{mol} \mathrm{m}{ }^{-2}\right)\end{array}$ & $A_{\min }\left(\AA^{2}\right)$ & $\begin{array}{l}\gamma_{\mathrm{CMC}} \\
\left(\mathrm{mN} \mathrm{m}^{-1}\right)\end{array}$ & $\begin{array}{l}\Pi_{\mathrm{CMC}} \\
\left(\mathrm{mN} \mathrm{m}^{-1}\right)\end{array}$ & $p C_{20}$ \\
\hline \multicolumn{11}{|l|}{$\mathrm{C}_{10} \operatorname{mimBr}$} \\
\hline & 308.15 & 30.78 & -38.42 & -3.93 & -34.49 & 1.68 & 98.92 & 45.15 & 26.82 & 1.70 \\
\hline & 318.15 & 31.60 & -39.53 & 0.15 & -39.68 & 1.57 & 105.8 & 41.29 & 30.68 & 1.63 \\
\hline 14 & 298.15 & 22.32 & -38.77 & -9.22 & -29.55 & 105.53 & 38.50 & 33.47 & 38.50 & 105.53 \\
\hline \multirow[t]{3}{*}{30} & 298.15 & 19.89 & -39.34 & -18.42 & -20.92 & 108.31 & 36.02 & 35.95 & 36.02 & 108.31 \\
\hline & 308.15 & 22.55 & -40.02 & -11.89 & -28.13 & 114.96 & 36.00 & 34.38 & 37.59 & 114.96 \\
\hline & 318.15 & 24.38 & -40.91 & -5.77 & -35.13 & 121.99 & 34.68 & 34.06 & 37.91 & 121.99 \\
\hline \multicolumn{11}{|l|}{$\mathrm{C}_{12} \mathrm{mimBr}$} \\
\hline 0 & 298.15 & 9.19 & -43.17 & -35.96 & -7.21 & 1.80 & 92.49 & 46.71 & 25.26 & 2.35 \\
\hline & 318.15 & 10.31 & -45.45 & -26.28 & -19.18 & 1.68 & 98.69 & 36.72 & 35.25 & 2.83 \\
\hline \multirow[t]{3}{*}{8} & 298.15 & 8.90 & -43.33 & -32.39 & -10.94 & 1.86 & 89.47 & 37.41 & 34.56 & 2.92 \\
\hline & 308.15 & 9.51 & -44.44 & -28.03 & -16.41 & 1.83 & 90.50 & 34.78 & 37.19 & 2.92 \\
\hline & 318.15 & 9.90 & -45.67 & -23.95 & -21.72 & 1.81 & 91.49 & 34.53 & 37.44 & 2.90 \\
\hline \multicolumn{11}{|l|}{$\mathrm{C}_{16} \mathrm{mimBr}$} \\
\hline \multirow[t]{3}{*}{0} & 298.15 & 0.610 & -56.61 & -33.36 & -23.24 & 2.00 & 83.14 & 44.53 & 27.44 & 3.42 \\
\hline & 308.15 & 0.631 & -58.34 & -29.01 & -29.33 & 1.83 & 90.50 & 42.29 & 29.68 & 3.40 \\
\hline & 318.15 & 0.637 & -60.18 & -24.93 & -35.25 & 1.61 & 103.33 & 41.74 & 30.23 & 3.34 \\
\hline \multirow[t]{3}{*}{0.02} & 298.15 & 0.601 & -56.69 & -25.33 & -31.36 & 2.22 & 74.83 & 42.64 & 29.33 & 3.48 \\
\hline & 308.15 & 0.624 & -58.40 & -20.97 & -37.42 & 2.05 & 81.02 & 41.25 & 30.72 & 3.40 \\
\hline & 318.15 & 0.632 & -60.22 & -16.89 & -43.33 & 1.76 & 94.44 & 40.39 & 31.58 & 3.34 \\
\hline
\end{tabular}

tetrabutylammonium dodecylsulfate (TBADS) in aqueous solutions of glycine with sodium dodecylsulfate (SDS). ${ }^{22}$

3.2.2 Surface properties of IL-surfactant mixtures. The molecules of the IL-surfactant mixture adsorbed at the interface, even at low surfactant concentration, cause the reduction of the surface tension of water. Possible interactions with the surfactant adsorption are hydrophobic effect, electrostatic interaction, van der Waals interaction, hydrogen bonding, ${ }^{12,14,38,39}$ etc.

It is known that the hydrophobicity of surfactants increases with increasing hydrophobic chain length. Thus the surfactant monomers are more likely to escape the solvent into the airliquid interface, so that the hydrophilic head group in the water is pulled by the hydrophobic hydrocarbon chain away from the solvent. Therefore, the parameter of $\Pi_{\mathrm{CMC}}$ in Table 2 reflects that the surface tension of the $\mathrm{C}_{16} \mathrm{mimBr} / \mathrm{CTAB}$ mixture is reduced more than that of $\mathrm{C}_{12} \mathrm{mimBr} / \mathrm{CTAB}$ when $\mathrm{CTAB}$ monomers get into solution. While in the $\mathrm{C}_{10} \mathrm{mimBr} / \mathrm{CTAB}$ system, the CTAB micelles almost have no effect in reducing the surface tension of water according to the $\Pi_{\mathrm{CMC}}$ listed in Table 2.

The adsorption efficiency $\left(p C_{20}\right)$ of the surfactant molecules at the air-solution interface can be obtained in terms of the surface tension data. For $\mathrm{C}_{n} \mathrm{mimBr} / \mathrm{CTAB}$ mixtures, it can be easily observed that $p C_{20}$ values increase when increasing the $\mathrm{CTAB}$ content, showing superior adsorption efficiency. ${ }^{14}$ However, $p C_{20}$ decreasing with the increment of temperature suggests that elevated temperature is not favorable for micellization, corresponding to CMC trends. It is noted that the $\mathrm{C}_{10} \mathrm{mimBr} / \mathrm{CTAB}$ mixtures exhibit increased $p C_{20}$ values in the case of CTAB micelles, different from the decrease in the $\mathrm{C}_{16} \mathrm{mimBr} / \mathrm{CTAB}$ mixtures with CTAB monomer added into the solution. So the self-assembled behaviors of surfactant molecules in mixture solution are influenced by the CTAB molecule state at different temperatures.

Additionally, the maximum surface excess concentration $\left(\Gamma_{\max }\right)$ is close to $p C_{20}$, and the minimum area of a single surfactant molecule $\left(A_{\min }\right)$ is also estimated in Table 1 . Like the temperature effect of $p C_{20}$ mentioned above, it is shown that the higher the temperature, the greater the value of $A_{\min }$ in the presence of CTAB monomer in solution, while $A_{\min }$ gradually decreases with temperature when CTAB micelles are present in solution. By comparing the data for the three ILs with the same counter ion, it was found that $A_{\min }$ per adsorbed ionic liquid at the air/water interface decreases with the length of the alkyl 
chain and it implies that the longer the alkyl chains, the more compact the monolayers at the air/water interface. ${ }^{40}$ Certainly, the values of $\Gamma_{\max }$ have the corresponding opposite trend. ${ }^{14}$ All these parameter changes can be thought to be related to enhanced molecular motion at elevated temperature, thus leading to fewer surfactant molecules being adsorbed at the airwater interface. ${ }^{1}$ With an excess of CTAB, more CTAB molecules may participate in surface adsorption of mixture solution, besides self-assembly behavior, for example in the $\mathrm{C}_{10} \mathrm{mimBr}$ / CTAB mixtures.

3.2.3 Thermodynamic functions of micellization. By applying eqn (8)-(10), the standard free energy change $\left(\Delta G_{\mathrm{m}}\right)$, standard enthalpy $\left(\Delta H_{\mathrm{m}}\right)$ and standard entropy change $\left(\Delta S_{\mathrm{m}}\right)$ associated with the micelle formation were also calculated. In the case of ionic surfactants, according to eqn (5)-(8), the standard free energy change as well as other thermodynamic functions of aggregate formation at different temperatures may be calculated using $X_{\mathrm{CMC}}{ }^{4}$ because the measurement of the degree of dissociation $(\alpha)$ may be omitted. Using eqn (8)-(10), the thermodynamic parameters of the CTAB with $\mathrm{C}_{n} \mathrm{mimBr}$ mixed system at different $\mathrm{CTAB}$ concentrations are given in Table 2. Comparison to the results from the conductivity measurement (Fig. 4) showed that three thermodynamic parameters with temperature and $\mathrm{CTAB}$ concentration displayed a similar change, and here an entropy-driven process can also be clearly observed from the data in Table 2, which indicates a CMC value a little smaller than that determined by the conductivity measurement. The difference in the two results is mainly based on applying different eqn (5) and (8).

$$
\Delta G_{\mathrm{m}}=2 R T \ln X_{\mathrm{CMC}}
$$

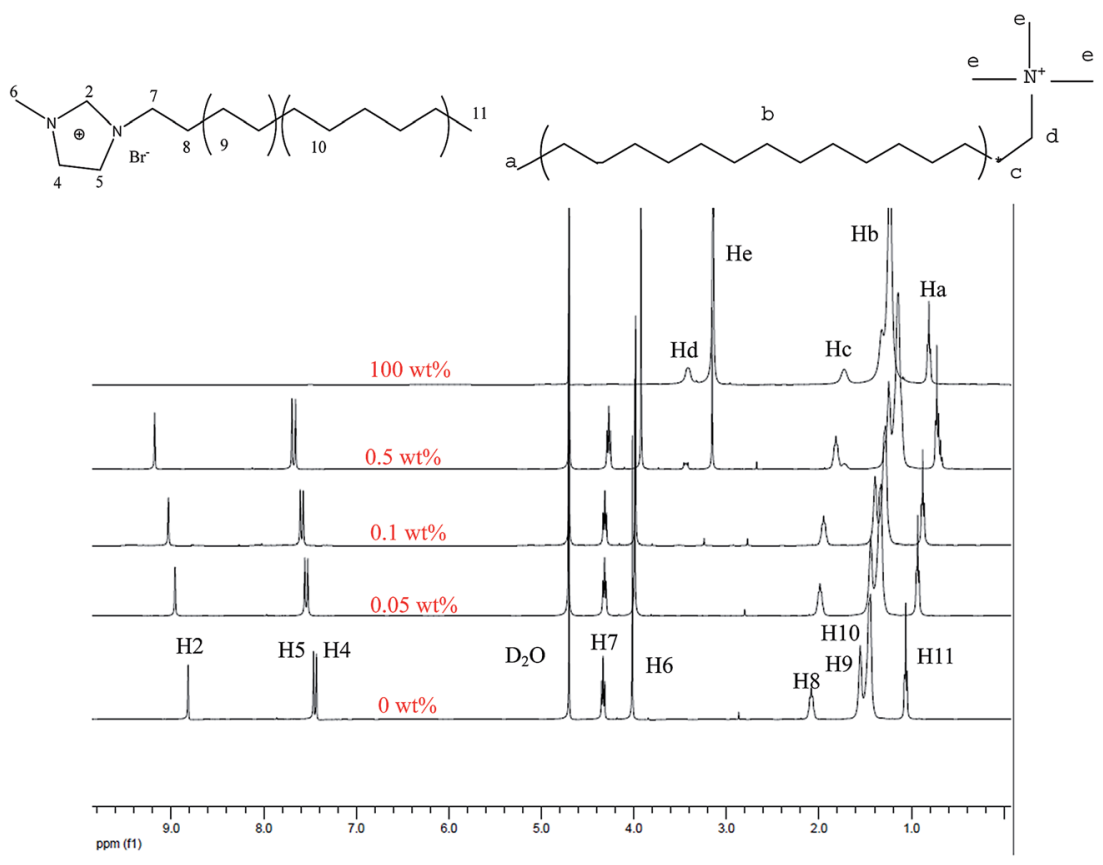

$$
\begin{gathered}
\Delta H_{\mathrm{m}}=\mathrm{d}\left(\frac{\Delta G_{\mathrm{m}}}{T}\right) / \mathrm{d}\left(\frac{1}{T}\right) \\
\Delta S_{\mathrm{m}}=\frac{\Delta H_{\mathrm{m}}-\Delta G_{\mathrm{m}}}{T}
\end{gathered}
$$

For the conductivity of the mixture solution, the degree of counter-ion dissociation implies that a fraction of the counterions on the micelle surface are confined, and the formed micelles can also carry less charge to transport than the free ions. ${ }^{4}$ Consequently, the $\alpha$ value should change with the number and state of the ionic surfactants in the mixture solution, so it is not a constant " 2 " in eqn (8), which is an extreme case of $\alpha \approx 0$. Theoretically, eqn (5) is more reasonable and accordingly thermodynamic functions shown in Fig. 4 seem to be more convincing.

\subsection{FT-IR analysis}

According to experimental method mentioned above, FT-IR spectra of $\mathrm{C}_{10} \mathrm{mimBr}, \mathrm{CTAB}$ and their mixture studied in the present work were recorded, as shown in Fig. S3. $\dagger$ The FT-IR spectra of the mixture showed the apparent change with comparison to the pure components. The $\mathrm{C}-\mathrm{H}$ stretching $\left(\mathrm{C}_{4} \mathrm{H}\right.$, $\mathrm{C}_{5} \mathrm{H}$ and $\mathrm{C}_{2} \mathrm{H}$ ) of the imidazole ring of IL can be observed at 3050-3200 $\mathrm{cm}^{-1},{ }^{3,41}$ but becomes weaker after interaction with $\mathrm{CTAB}$ in the mixture. It is worth noting that a band located at $1572 \mathrm{~cm}^{-1}$ (C-N stretching) for the IL was weaker in the mixture than in the pure IL. Moreover, the characteristic peak of $\mathrm{C}-\mathrm{H}$ plane deformation vibration in the imidazole ring significantly moves from $1174 \mathrm{~cm}^{-1}$ to $1168 \mathrm{~cm}^{-1}$. This is evidence of the electrostatic effect between the head groups of IL and CTAB molecules. On the other hand, the band between 2800 and

Fig. $8{ }^{1} \mathrm{H}$ NMR spectra of $\mathrm{C}_{10} \mathrm{mimBr}$ in $\mathrm{D}_{2} \mathrm{O}$ solution with different wt\% of CTAB. 
$3050 \mathrm{~cm}^{-1}$ assigned to the asymmetric and symmetric $\mathrm{C}-\mathrm{H}$ stretching vibration of the methyl groups was seen in $\mathrm{C}_{10}$ mimBr, CTAB and their mixture spectra, ${ }^{42}$ but the slight change corresponds to the hydrophobic interactions between the hydrocarbon chain of the surfactant CTAB and IL cation.

\section{$3.4 \quad{ }^{1} \mathrm{H}$ NMR}

The ${ }^{1} \mathrm{H}$ NMR spectra were recorded to understand the various intermolecular interactions present among IL and CTAB molecules in aqueous solutions. In this part, the effect of CTAB on the NMR microenvironment of the $\mathrm{C}_{n}$ mimBr was investigated and all protons of $\mathrm{C}_{n} \mathrm{mimBr}$ in the mixed system were analyzed. The ${ }^{1} \mathrm{H}$ NMR spectrum of the IL in Fig. 8 reveals nine characteristic peaks. It can be seen that the characteristic proton signals of $\mathrm{Ha}-\mathrm{He}$ from CTAB gradually become higher and higher with increasing CTAB. It is well known that the chemical shifts of IL protons move upfield or downfield depend on the shielding and deshielding effects of surrounding groups. The greater the electronic density, the greater the shielding effect. Owing to addition of CTAB, all upfield shifts in the tail chain of the IL proton signals of $\mathrm{H} 7-11$ reflect the hydrophobic interactions present among the hydrophobic chain of the surfactant and alkyl chains of the IL cation, which led to shielding of the surrounding protons. ${ }^{12}$

In addition, the evident downfield shift of the head protons $(\mathrm{H} 2,4,5)$ of the IL was observed. This may be interpreted that after the addition of $\mathrm{CTAB}$, increased counterion dissociation from CTAB into solution leads to a stronger electrostatic attractive effect between the $\mathrm{Br}^{-}$and the imidazolium cation than electrostatic repulsion, thus the lower electronic density of the imidazolium cation results in the signals of $\mathrm{H} 2,4$, and 5 shifting toward lower field. ${ }^{27}$ However, an upfield shift was also observed for protons of the imidazole ring (H6), but not significant in comparison with the tail region protons (H8-11) because the magnetic environment of proton H6 located at the micellar surface would not be changed so remarkably after addition of CTAB.

\section{Conclusion}

The present study presents the surface and thermodynamic properties of the $\mathrm{C}_{n}$ mimBr/CTAB surfactants system in aqueous media using surface tension and conductivity methods. For all the systems, the surface activity and CMC values of the $\mathrm{C}_{n^{-}}$ $\operatorname{mimBr} / \mathrm{CTAB}(n=10,12,16)$ systems are highly dependent on the CTAB concentration and temperature. The CMC values systematically decreased with the CTAB concentration and an inverse trend was observed when temperature was increased; such dependence is analogous with that of conventional ionic surfactants. It was further perceived that adsorption proficiency decreased at high CTAB monomer concentrations, while the opposite result was also observed in the presence of CTAB micelles. On the other hand, the minimum area per adsorbed molecule decreased sharply with CTAB concentration, indicating that compact mono-layers are formed at the air/water interface and facilitate the formation of well-defined aggregates in the bulk. The value of surface excess concentration $\left(\Gamma_{\max }\right)$ implies that the adsorption of IL in the mixture system decreases with increasing CTAB content, while opposite trends were observed in other surfactant systems. For all the mixed systems, the negative $\Delta G_{\mathrm{m}}$ presented a decrease with temperature and $\mathrm{CTAB}$ concentration, suggesting that all micellization processes were spontaneous. The $\Delta H_{\mathrm{m}}$ and $\Delta S_{\mathrm{m}}$ values for all the mixed systems were dependent on the CTAB molecule state, as the CTAB monomer and micelle led to an opposite change trend of $\Delta H_{\mathrm{m}}$ and $\Delta S_{\mathrm{m}}$ for the mixed system. FT-IR and NMR data provide evidence of the interaction between the IL and CTAB molecules in the mixture system to best understand the cause of the final results. By comparison of the traditional surfactants with CTAB addition into ionic liquid surfactants to form a surfactants mixture, it is important to reveal the higher surface activity. Therefore, similar to classical cationic surfactants, the active surface of such a surfactant mixture forms well defined aggregates at the air/water interface in bulk solution and has potential for the development of novel green surface-active agents. It is clear that the present investigations provide a fundamental understanding of the solution properties of the binary system. Our work herein will also be useful for designing separation technologies and would be further useful in developing new technologies for applying ILs to colloid and interfacial science chemical engineering fields as well.

\section{Conflicts of interest}

All authors declare no financial/commercial conflicts of interest.

\section{Acknowledgements}

The authors are grateful for the open project of Jiangsu Key Laboratory for Bioresources of Saline Solis (JKLBS2016012), and Jiangsu Province College Students' Innovative Training Project (201610324044x) and a Project Funded by the Excellent Specialties Program Development of Jiangsu Higher Education Institutions, Project (No. PPZY2015B113).

\section{References}

1 B. Dong, X. Y. Zhao, L. Q. Zheng, J. Zhang, N. Li and T. Inoue, Colloids Surf., A, 2008, 317, 666-672.

2 J. Saien and S. Asadabadi, Fluid Phase Equilib., 2015, 386, 134-139.

3 K. Li and T. Kobayashi, Ultrason. Sonochem., 2016, 28, 39-46. 4 X. W. Li, Y. A. Gao, J. Liu, L. Q. Zheng, B. Chen, L. Z. Wu and C. H. Tung, J. Colloid Interface Sci., 2010, 343, 94-101.

5 Y. Li, M. Zhang, Q. Liu and H. Su, J. Chem. Thermodyn., 2013, 66, 80-87.

6 P. Bharmoria, T. Singh and A. Kumar, J. Colloid Interface Sci., 2013, 407, 361-369.

7 J. Luczak, M. Paszkiewicz, A. Krukowska, A. Malankowska and A. Zaleska-Medynska, Adv. Colloid Interface Sci., 2016, 230, 13-28. 
8 S. Livi, J. Duchet-Rumeau, T. N. Pham and J. F. Gerard, J. Colloid Interface Sci., 2010, 349, 424-433.

9 F. Comelles, I. Ribosa, J. J. Gonzalez and M. T. Garcia, Colloids Surf., A, 2015, 484, 136-143.

10 O. Nacham, A. Martín-Pérez, D. J. Steyer, M. J. TrujilloRodríguez, J. L. Anderson, V. Pino and A. M. Afonso, Colloids Surf., A, 2015, 469, 224-234.

11 S. Chabba, S. Kumar, V. K. Aswal, T. S. Kang and R. K. Mahajan, Colloids Surf., A, 2015, 472, 9-20.

12 S. Zhang, Y. Gao, B. Dong and L. Zheng, Colloids Surf., A, 2010, 372, 182-189.

13 K. Thakkar, B. Bharatiya, D. O. Shah, D. Ray, V. K. Aswal and P. Bahadur, Colloids Surf., A, 2015, 484, 547-557.

14 T. Inoue, K. Kawashima and Y. Miyagawa, J. Colloid Interface Sci., 2011, 363, 295-300.

15 J. Liu, L. Zheng, D. Sun and X. Wei, Colloids Surf., A, 2010, 358, 93-100.

16 K. D. Danov, P. A. Kralchevsky and K. P. Ananthapadmanabhan, Adv. Colloid Interface Sci., 2014, 206, 17-45.

17 D. Bhatt, K. Maheria and J. Parikh, J. Chem. Thermodyn., 2014, 74, 184-192.

18 J. M. Padró and M. Reta, J. Mol. Liq., 2016, 213, 107-114.

19 I. J. Warke, K. J. Patil and S. S. Terdale, J. Chem. Thermodyn., 2016, 93, 101-114.

20 L. M. Bergstrom and V. M. Garamus, J. Colloid Interface Sci., 2012, 381, 89-99.

21 A. Pal and A. Pillania, J. Mol. Liq., 2015, 212, 818-824.

22 S. Chauhan, A. Kumar, M. Kaur and M. S. Chauhan, J. Surfactants Deterg., 2017, 20, 1129-1139.

23 J. Łuczak, A. Latowska and J. Hupka, Colloids Surf., A, 2015, 471, 26-37.

24 V. G. Rao, S. Mandal, S. Ghosh, C. Banerjee and N. Sarkar, J. Phys. Chem. B, 2012, 116, 13868-13877.
25 X. Wang, J. Liu, L. Yu, J. Jiao, R. Wang and L. Sun, J. Colloid Interface Sci., 2013, 391, 103-110.

26 S. Chauhan, V. Sharma, K. Singh, M. S. Chauhan and K. Singh, J. Mol. Liq., 2016, 222, 67-76.

27 A. Pal and A. Pillania, Fluid Phase Equilib., 2015, 389, 67-73. 28 A. Pal and A. Pillania, J. Mol. Liq., 2015, 209, 6-13.

29 P. Wang, X.-F. Guo, L.-H. Lia, C.-M. Ma and L. Qian, Chin. J. Appl. Chem., 2013, 30, 840-845.

30 A. Pal and S. Chaudhary, J. Mol. Liq., 2015, 207, 67-72.

31 A. Pal and A. Pillania, Fluid Phase Equilib., 2016, 412, 115122.

32 S. Chauhan, Atika, K. Singh, K. Singh, M. Kaur and M. S. Chauhan, J. Mol. Liq., 2017, 242, 1066-1074.

33 A.-D. M. Sørensen, K. S. Lyneborg, P. Villeneuve and C. Jacobsen, J. Funct. Foods, 2015, 18, 959-967.

34 B. Sohrabi, S. Eivazzadeh, A. Sharifi and R. Azadbakht, J. Mol. Liq., 2015, 211, 754-760.

35 S. Chauhan and M. Kaur, J. Surfactants Deterg., 2017, 20, 599607.

36 S. Chauhan, S. Kumari and K. Singh, J. Chem. Thermodyn., 2017, 105, 337-344.

37 G.-Y. Wang, Y.-Y. Wang and X.-H. Wang, J. Mol. Liq., 2017, 232, 55-61.

38 M. N. Garaga, M. Nayeri and A. Martinelli, J. Mol. Liq., 2015, 210, 169-177.

39 H. Y. He, H. Chen, Y. Z. Zheng, S. J. Zhang and Z. W. Yu, Chem. Eng. Sci., 2015, 121, 169-179.

40 N. V. Sastry, N. M. Vaghela and V. K. Aswal, Fluid Phase Equilib., 2012, 327, 22-29.

41 Y. Jiang, L. Wang, L. Zhang, T. Wang, Y. Zhou, C. Ding, R. Yang, X. Wang and L. Yu, Int. J. Biol. Macromol., 2015, 79, 533-541.

42 C. Azémard, C. Vieillescazes and M. Ménager, Microchem. J., 2014, 112, 137-149. 